\title{
Taxifolin suppresses the malignant progression of gastric cancer by regulating the AhR/CYP1A1 signaling pathway
}

\author{
JIEBIN XIE ${ }^{1,2}$, YUESHAN PANG ${ }^{3}$ and XIAOTING WU ${ }^{1}$ \\ ${ }^{1}$ Department of Gastrointestinal Surgery, West China Hospital, Sichuan University, Chengdu, Sichuan 610041; \\ ${ }^{2}$ Department of Gastrointestinal Surgery, Affiliated Hospital of North Sichuan Medical College; \\ ${ }^{3}$ Department of Geriatrics, The Second Clinical Medical College of North Sichuan Medical College, \\ Nanchong Central Hospital, Nanchong, Sichuan 637000, P.R. China
}

Received February 7, 2021; Accepted June 11, 2021

DOI: $10.3892 / \mathrm{ijmm} .2021 .5030$

\begin{abstract}
The development of novel approaches for the treatment of gastric cancer is of utmost importance. Taxifolin (Tax) has been reported to possess biological activities against a number of types of cancer. The objective of the present study was to examine the effects of Tax on gastric cancer and to explore its potential mechanisms of action. For this purpose, AGS and NCI-N87 cells, as well as BALB/c mice with gastric cancer cell-derived tumors were treated with Tax. Cell Counting Kit- 8 and colony formation assays were performed to detect cell viability and proliferation, respectively. Wound-healing and Transwell assays were also conducted to determine the cell migratory and invasive abilities, respectively. Western blot analysis was performed to determine protein expression in vitro and in vivo. The results revealed that Tax significantly inhibited the viability, proliferation, migration and invasion of gastric cancer cells through the aryl hydrocarbon receptor (AhR)/cytochrome P450 1A1 (CYP1A1) signaling pathway. SB203580, an AhR agonist, partly abolished the inhibitory effects of Tax on gastric cancer cell viability, proliferation, migration and invasion. In addition, Tax also suppressed tumor growth in vivo. Collectively, the present study demonstrated that Tax significantly suppressed the tumor characteristics of gastric cancer. Tax may thus prove to be a potential therapeutic strategy for gastric cancer.
\end{abstract}

\section{Introduction}

Gastric cancer is the fifth most common malignancy and the third most common cause of cancer-related mortality worldwide, with over 1 million estimated new cases annually and 784,000 deaths globally in 2018, prompting the World Health

Correspondence to: Professor Xiaoting Wu, Department of Gastrointestinal Surgery, West China Hospital, Sichuan University, 37 Guoxue Lane, Chengdu, Sichuan 610041, P.R. China

E-mail: wuxiaotingwxt@126.com

Key words: taxifolin, gastric cancer, invasion, aryl hydrocarbon receptor, cytochrome $\mathrm{P} 450$ 1A1
Organization to declare it a public health concern $(1,2)$. Gastric cancer is a multi-step and multi-factorial disease. The efficacy of gastric cancer treatment is dependent on the stage of the tumor. Patients with early-stage gastric cancer, who receive radical surgery, have a favorable 5-year survival rate; however, the majority of patients are diagnosed at an advanced stage due to the inability of early detection, and the 5-year survival rate of these patients is generally poor (3). Hence, a novel therapeutic agent, as well as an improved understanding of the molecular mechanisms underlying gastric cancer, are urgently required in order to improve patient prognosis and the survival rate.

Taxifolin (Tax), also known as dihydroquercetin (3,5,7,3,4-pentahydroxy flavanone), is a flavonoid naturally occurring in milk thistle, onion, Douglas fir bark and French maritime pine bark, which has been reported to possess multiple biological activities in the management of oxidative stress, inflammation, microbial infections, liver and cardiovascular disorders, as well as tumors (4). Tax has also been identified as a potential antitumor agent in different types of cancer, such as osteosarcoma, colorectal, breast and lung cancer (5-8). For example, Razak et al (6) demonstrated that Tax induced cytotoxicity and cell cycle arrest in colorectal cancer cells and hampered the tumor growth of HCT116-derived xenografts in mice. Li et al (5) reported that Tax not only had the potential to inhibit the proliferation, migration and invasion of breast cancer cells in vitro, but also hindered the growth of primary tumors and reduced the lung metastasis of breast cancer in vivo. However, to the best of the authors' knowledge, no research performed to date has reported the antitumor effects of Tax in gastric cancer.

In the present study, the effects of Tax were examined on two gastric cancer cell lines, AGS and NCI-N87 cells in vitro, and tumor-bearing mice in vivo, and the potential regulatory mechanisms of Tax were further investigated. The present study was conducted in accordance with the ARRIVE guidelines checklist (9).

\section{Materials and methods}

Cell culture and treatment. Two human gastric cancer cell lines, AGS and NCI-N87, obtained from the American 
Type Culture Collection were cultured in DMEM (Gibco; Thermo Fisher Scientific, Inc.) supplemented with 10\% FBS (Gibco; Thermo Fisher Scientifc, Inc.) and 1\% penicillin/streptomycin (Gibco; Thermo Fisher Scientific, Inc.) in a humidified incubator with $5 \% \mathrm{CO}_{2}$ and $95 \%$ air at $37^{\circ} \mathrm{C}$. For treatment, Tax was obtained from Shanghai Huicheng Technology, Ltd. The AGS and NCI-N87 cells were treated with increasing concentrations of $\operatorname{Tax}(1,3,10,30$ and $100 \mu \mathrm{M})$ for $48 \mathrm{~h}$. In addition, the aryl hydrocarbon receptor (AhR) agonist SB203580 (10 $\mu \mathrm{M}$; Sigma-Aldrich; Merck KGaA) was applied for further treatment.

Cell Counting Kit-8 (CCK-8) assay. Cell viability was assessed using a CCK-8 assay (Beyotime Institute of Biotechnology). The AGS and NCI-N87 cells were cultured in 96-well plates $\left(1 \times 10^{4}\right.$ cells/well) for $24 \mathrm{~h}$, and were then treated with various concentrations of $\operatorname{Tax}(1,3,10,30$ and $100 \mu \mathrm{M})$ for a further $48 \mathrm{~h}$. Then, $10 \mu \mathrm{l}$ CCK- 8 reagent was added to each well, and the cells were incubated at $37^{\circ} \mathrm{C}$ for $3 \mathrm{~h}$. The absorbance at $450 \mathrm{~nm}$ was detected using a microplate reader (ELx800; BioTek Instruments, Inc.). The results are presented as a relative percentage of the untreated control cells.

Colony formation assay. The cell proliferative ability was assessed using a colony formation assay. Cells were seeded into six-well plates at a density of 500 cells/well. After being subjected to the Tax $(100 \mu \mathrm{M})$ treatment with or without SB203580 $(10 \mu \mathrm{M})$ treatment, cells were incubated in a $5 \% \mathrm{CO}_{2}$ incubator at $37^{\circ} \mathrm{C}$ for 2 weeks. Thereafter, the cells were fixed with methanol for $10 \mathrm{~min}$ at room temperature and stained with $0.5 \%$ crystal violet for a further $10 \mathrm{~min}$ at room temperature. Images were captured under a light microscope (magnification, $\mathrm{x} 10$ ), and colonies containing $>50$ cells were counted.

Wound-healing assay. The cell migratory ability was assessed using a wound-healing assay. The cells were re-suspended with serum-free medium and added to 24-well plates for a $24-\mathrm{h}$ incubation at $37^{\circ} \mathrm{C}$. Upon reaching $100 \%$ confluency, a scratch was subsequently generated in the cell monolayer using a sterile micropipette tip. The cells were then incubated in serum-free medium containing Tax $(100 \mu \mathrm{M})$ with or without SB203580 $(10 \mu \mathrm{M})$ at $37^{\circ} \mathrm{C}$ for $24 \mathrm{~h}$. The wound width at 0 and $24 \mathrm{~h}$ was captured using a light microscope (magnification, x100).

Transwell assay. The cell invasive ability was assessed using a Transwell assay with a 24-well Transwell plate with pore size of $8-\mu \mathrm{m}$ (EMD Millipore) precoated with BD Matrigel (BD Biosciences) at $37^{\circ} \mathrm{C}$ for $1 \mathrm{~h}$. The cells were suspended in serum-free medium and added to the upper chamber ( $3 \times 10^{4}$ cells/well) of the 24 -well Transwell plate, followed by a 24-h incubation of Tax $(100 \mu \mathrm{M})$ with or without SB203580 $(10 \mu \mathrm{M})$. Complete medium containing 10\% FBS was added to the lower chamber. Following $24 \mathrm{~h}$ of incubation at $37^{\circ} \mathrm{C}$, the non-invasive cells were removed using cotton swabs, and the invasive cells were fixed with $100 \%$ methanol for $10 \mathrm{~min}$ and stained with $0.1 \%$ crystal violet for $20 \mathrm{~min}$ at room temperature. Images were captured under a light microscope (magnification, x100).
Western blot analysis. Cells were lysed using RIPA lysis (Wuhan Boster Biological Technology, Ltd.) containing $1 \mathrm{mM}$ phenylmethylsulfonyl fluoride (PMSF). A BCA assay was used to determine the protein concentration. Equal amounts of protein (30 $\mu \mathrm{g} /$ lane) were separated by a $12 \%$ SDS-PAGE gel and transferred to PVDF membranes (EMD Millipore). Subsequently, the membranes were blocked in 5\% skimmed milk for $2 \mathrm{~h}$ at room temperature, and then incubated with corresponding primary antibodies against matrix metalloproteinase (MMP)2 (1:1,000; product code ab92536), MMP9 (1:1,000; product code ab38898), E-cadherin (1:1,000; product code ab231303), Zonula occludens-1 (ZO-1; 1:1,000; product code ab96587), N-cadherin (1:5,000; product code ab76011), Snail (1:1,000; product code ab180714), AhR (1:1,000; product code ab108518), cytochrome P450 1A1 (CYP1A1; 1:1,000; product code ab126887), Ki67 (1:1,000; product code ab16667), proliferating cell nuclear antigen, (PCNA; 1:1,000; product code ab92552) and GAPDH (1:1,000; product code ab8245) from Abcam at $4^{\circ} \mathrm{C}$ overnight. On the second day, the membranes were washed three times and incubated with HRP-conjugated goat anti-mouse (1:2,000; cat. no. sc-2005; Santa Cruz Biotechnology, Inc.) or goat anti-rabbit (1:2,000; product code ab97051; Abcam) antibodies for $2 \mathrm{~h}$ at room temperature. Bands were exposed by an enhanced chemiluminescence (ECL) kit (Beyotime Institute of Biotechnology) and analyzed using Image J software version 1.50 (National Institutes of Health).

In vivo experiments. A total of 24 male 6-week-old BALB/c null nude mice $(22 \pm 2 \mathrm{~g})$ were obtained from HFK Bioscience Co., Ltd. and housed at the Animal Care Facility of West China Hospital, Sichuan University (Chengdu, China) under a controlled temperature $\left(22 \pm^{\circ} \mathrm{C}\right)$ and humidity $(55 \pm 5 \%)$, with a 12-h light/dark cycle and free access to water and food. Prior to the operation, all mice were acclimatized for 1 week. Tumor xenografts in mice were established by injecting $1 \times 10^{6}$ AGS or NCI-N87 cells subcutaneously into the right flank region. After 5 days, the mice were randomly assigned into two groups ( $n=6$ for each group) and intraperitoneally injected with $25 \mathrm{mg} / \mathrm{kg}$ Tax twice weekly or an equal volume of saline, respectively. During this period, the tumor size and body weight of the mice were observed and recorded every 3 days. The allowed maximum diameter of the tumors was $1.5 \mathrm{~cm}$. At the end of the experiment (the 21st day), all the 24 mice were sacrificed by cervical dislocation under deep anesthesia (sodium pentobarbital intraperitoneal injection, $50 \mathrm{mg} / \mathrm{kg}$ ). After the cessation of the heartbeat and respiratory arrest of the mice was confirmed, the tumors were collected for measuring the weight and size, and frozen at $-80^{\circ} \mathrm{C}$ for use in subsequent western blot analysis. All animal experiments were performed in accordance with the Care and Use of Laboratory Animals established by the US National Institutes of Health (10), and were approved by the Ethics Committee of West China Hospital, Sichuan University (approval no. 2021-05).

Immunohistochemistry. The tumor specimens were dissected and fixed in $4 \%$ paraformaldehyde at $37^{\circ} \mathrm{C}$ for $48 \mathrm{~h}$. Then, the tissues were paraffin-embedded, and sectioned into 4- $\mu$ m-thick slices. The slices were deparaffinized, rehydrated and subjected to antigen retrieval. Subsequently, the slices were 


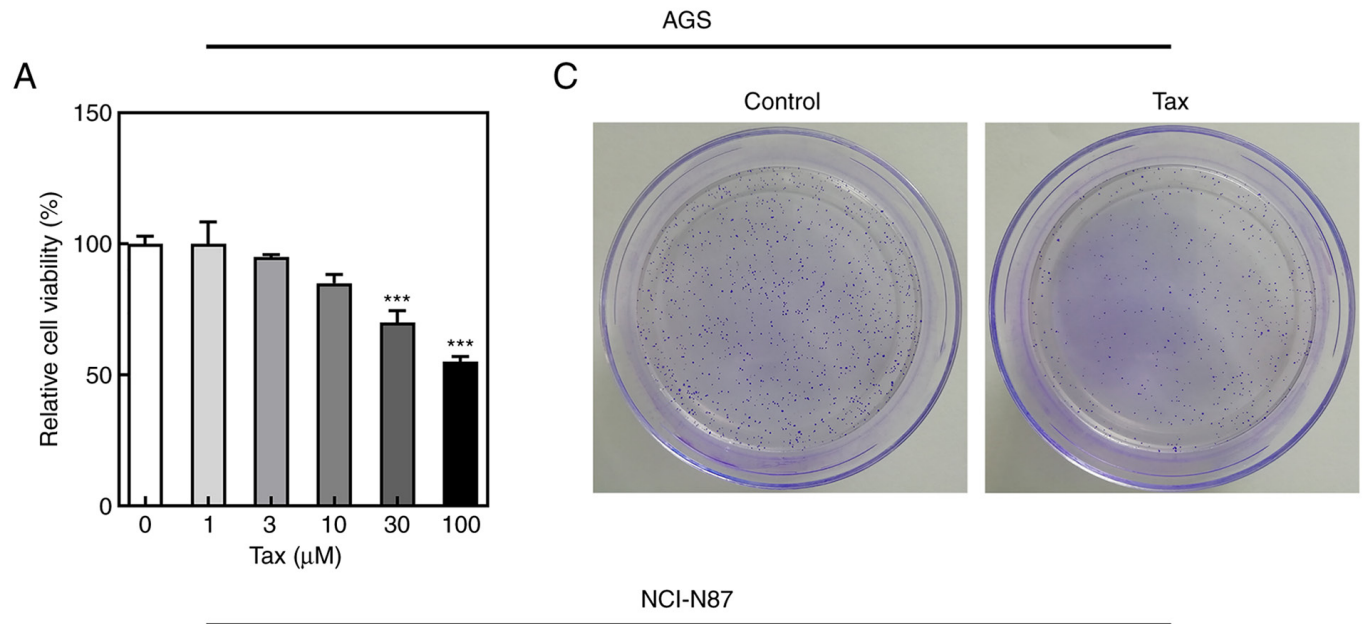

\section{B}

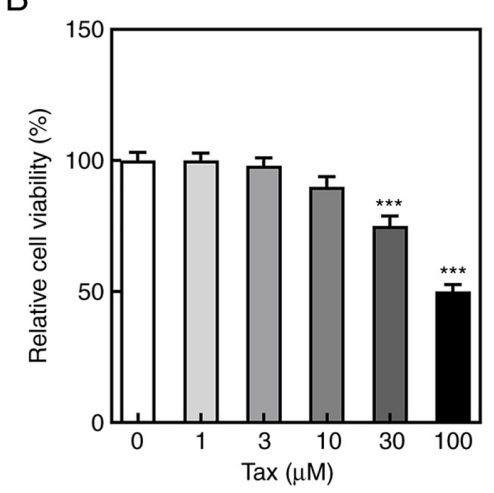

D

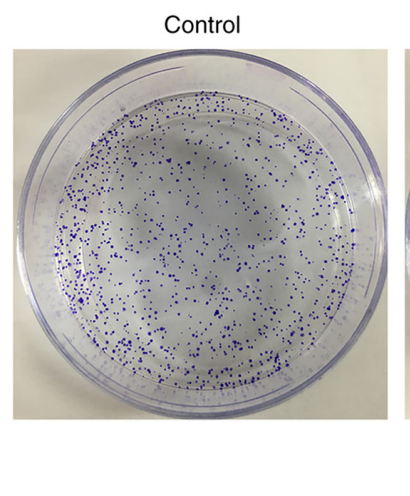

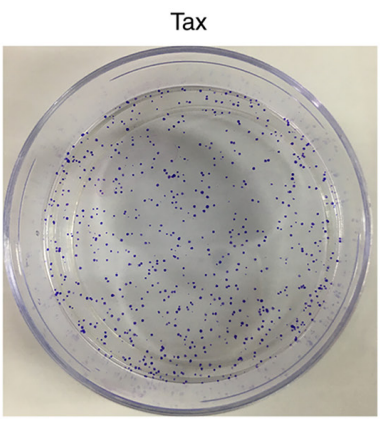

Figure 1. Effects of Tax on cell viability and proliferation in gastric cancer cells. (A and B) AGS and NCI-N87 cells were treated with different concentrations of Tax $(1,3,10,30$ and $100 \mu \mathrm{M})$, and the cell viability was detected using Cell Counting Kit- 8 assay. (C and D) AGS and NCI-N87 cells were treated with $100 \mu \mathrm{M}$ Tax and cell colony formation assays were performed. ${ }^{* * *} \mathrm{P}<0.001 \mathrm{vs}$. Tax $(0 \mu \mathrm{M})$. Tax, taxifolin.

blocked with 3\% bovine serum albumin (BSA; Sigma-Aldrich; Merck $\mathrm{KGaA}$ ) at room temperature for $30 \mathrm{~min}$, and incubated with anti-Ki67 antibody (1:200; product code ab16667; Abcam) at $4{ }^{\circ} \mathrm{C}$ overnight. After washing with PBS, the slices were incubated with HRP-conjugated goat anti-rabbit antibody (1:1,000; product code ab97051; Abcam). Slices were counterstained with hematoxylin for 2 min at room temperature and visualized with DAB (ZSJQ-BIO) under a light microscope (magnification, $\mathrm{x} 200)$.

Statistical analysis. SPSS 17.0 software (SPSS, Inc.) was used for statistical analysis and data are presented as the mean \pm SD. All data were determined from at least three independent experiments. A Student's unpaired t-test was performed for comparisons between two groups, and a one-way AVONA followed by a Tukey's post hoc test was performed for comparisons among more than two groups. $\mathrm{P}<0.05$ was considered to indicate a statistically significant difference.

\section{Results}

Effects of Tax on the viability and proliferation of gastric cancer cells. To examine the antitumor effects of Tax, two gastric cancer cell lines, AGS and NCI-N87, were treated with various concentrations of $\operatorname{Tax}(1,3,10,30$ and $100 \mu \mathrm{M})$. It was observed that treatment of the gastric cancer cells with Tax suppressed cell viability in a concentration-dependent manner (Fig. 1A and B). In subsequent experiments, $100 \mu \mathrm{M}$ Tax was selected to treat the AGS and NCI-N87 cells. A colony formation assay was performed to detect the changes in colony formation following Tax treatment. As revealed in Fig. 1C, Tax treatment decreased the number of cell colonies formed compared with the control group in both AGS and NCI-N87 cells. These results indicated that Tax had the ability to hinder cell proliferation.

Effects of Tax on the migratory and invasive abilities of gastric cancer cells. After demonstrating the inhibitory effects of Tax on the cell proliferative ability, the present study then determined whether Tax suppressed the cell migratory and invasive abilities. In a wound-healing assay, Tax treatment led to a lower 'healing' ability at $24 \mathrm{~h}$ in both the AGS and NCI-N87 cells. In addition, a Transwell assay revealed that Tax hindered the invasive ability of not only the AGS cells, but also the NCI-N87 cells (Fig. 2A-C). Moreover, the downregulated protein expression levels of MMP2 and MMP9 upon Tax treatment further demonstrated the role of Tax in gastric cancer (Fig. 2D). Furthermore, Tax treatment increased the protein expression levels of E-cadherin and ZO-1, and reduced the protein expression levels of $\mathrm{N}$-cadherin and Snail (Fig. 2E), indicating a potential inhibitory role of Tax in epithelial-mesenchymal transition (EMT) in gastric cancer. Therefore, these results indicated that Tax impeded the migratory and invasive abilities of gastric cancer cells by regulating EMT. 
A

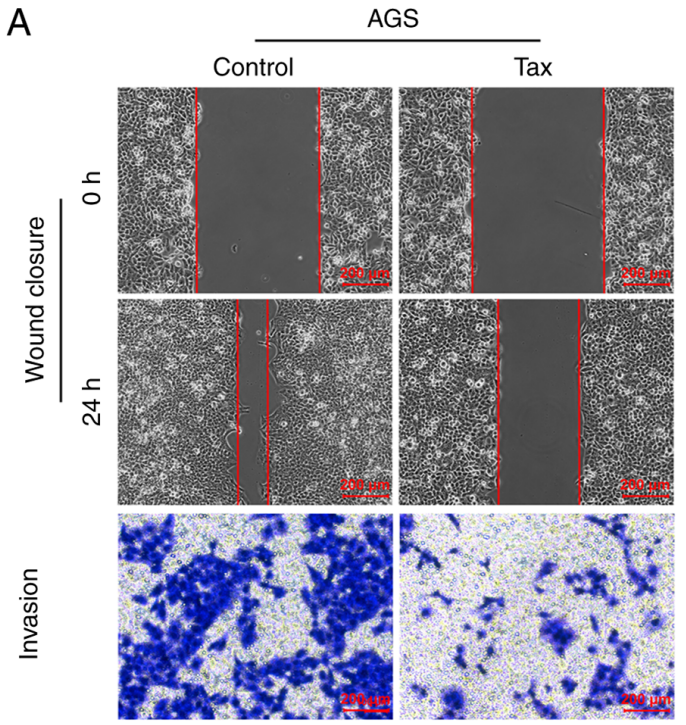

B

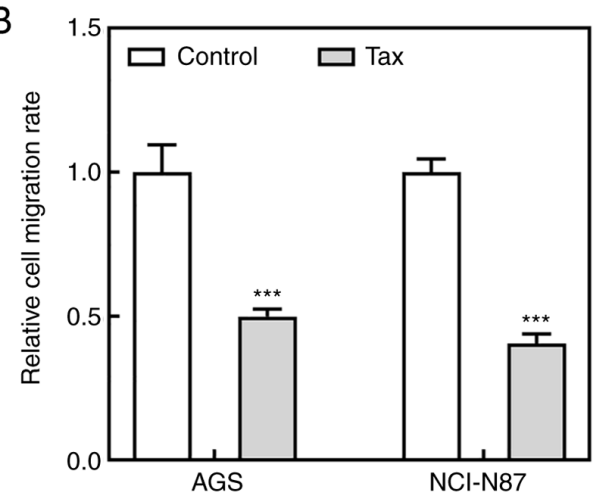

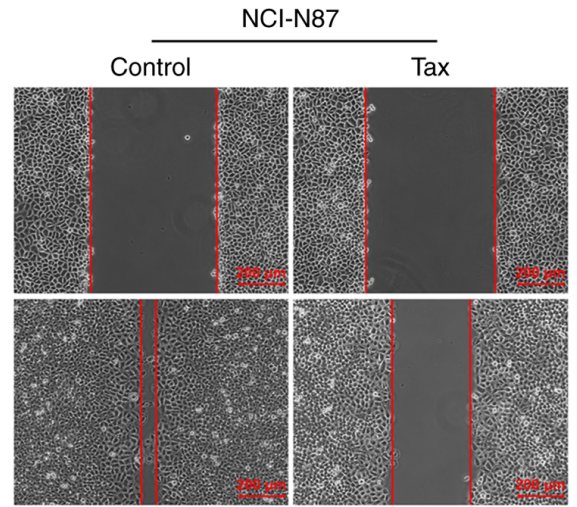

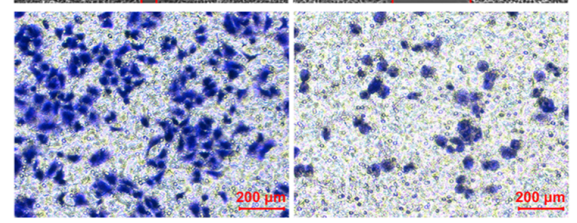

C

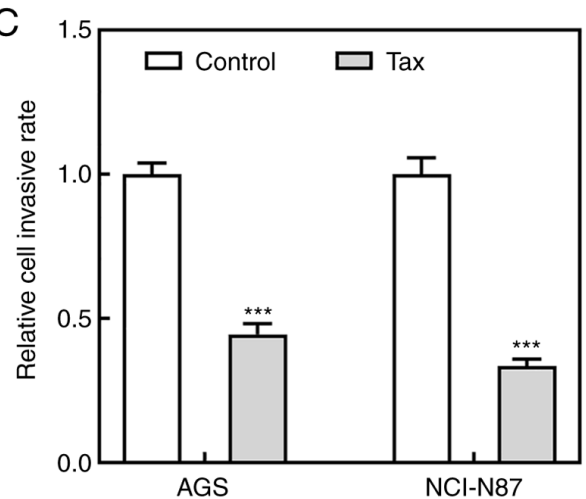

D

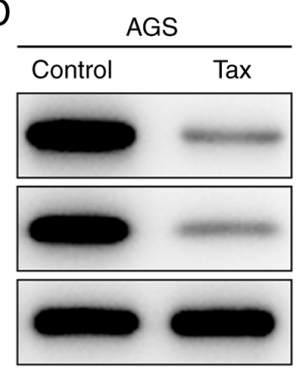

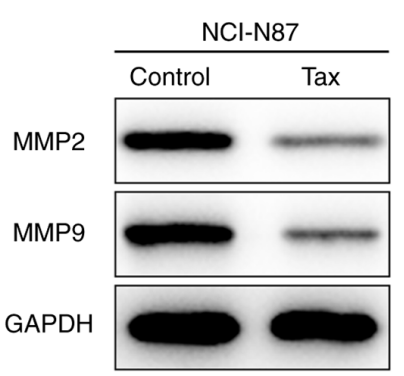

E

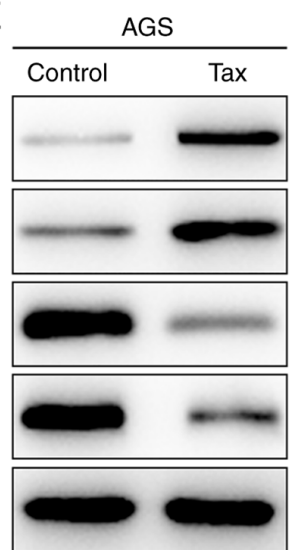

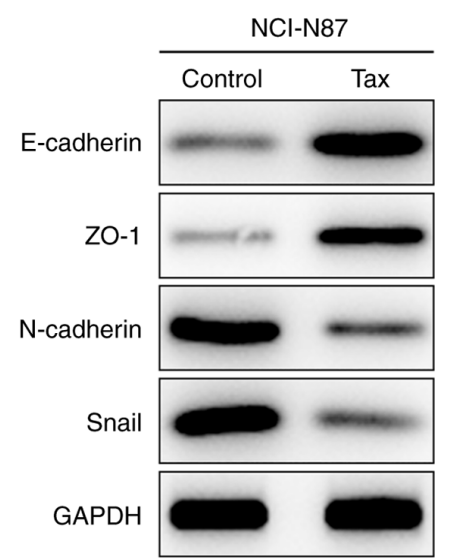

Figure 2. Effects of Tax on cell migration and invasion abilities in gastric cancer cells. (A) Wound-healing and Transwell assays were conducted to observe cell migration and invasion abilities, respectively. (B) The migration rate of each group was quantified. (C) The invasion rate of each group was quantified. (D and E) The protein expression of MMP2, MMP9, E-cadherin, ZO-1, N-cadherin and Snail was assessed by western blotting. ${ }^{* * *} \mathrm{P}<0.001$ vs. the control. Tax, taxifolin; MMP, matrix metalloproteinase; ZO-1, Zonula occludens-1.

Effect of Tax on the AhR/CYPIAl signaling pathway in gastric cancer cells. To elucidate the potential mechanisms underlying the protective role of Tax in gastric cancer, the effects of Tax on the AhR/CYP1A1 signaling pathway were examined. As revealed in Fig. 3A, Tax treatment significantly decreased the protein expression levels of AhR and CYP1A1 in AGS cells. A similar trend was observed in NCI-N87 cells (Fig. 3B). Therefore, these results indicated that Tax suppressed the activation of the AhR/CYP1A1 signaling pathway.
Effects of the AhR agonist, SB203580, on Tax-treated gastric cancer cells. Subsequently, to identify whether the protective role of Tax in gastric cancer was mediated via AhR/CYP1A1 signaling, the effects of the AhR agonist, SB203580, on Tax-treated gastric cancer cells were examined. Firstly, SB203580 was revealed to increase the protein expression levels of AhR and CYP1A1 in Tax-treated AGS or NCI-N87 cells (Fig. 4A and B). Subsequently, a series of cell biological behaviors were examined, as aforementioned. On the one 
A

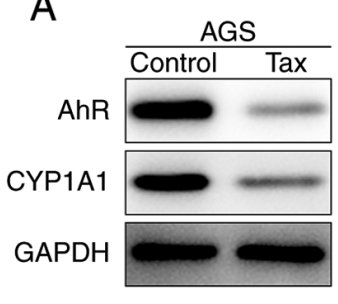

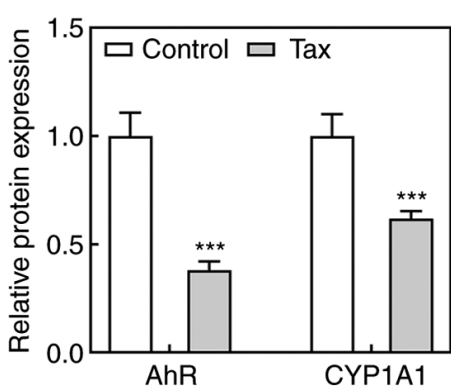

B

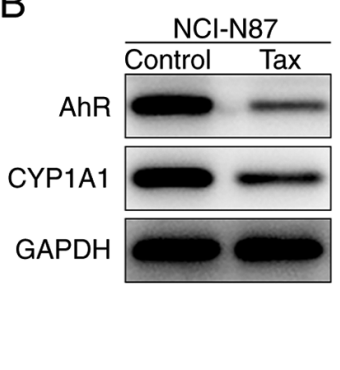

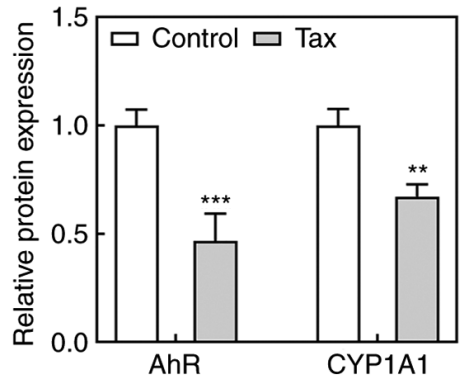

Figure 3. Effect of Tax on AhR/CYP1A1 signaling in gastric cancer cells. (A) In AGS cells, the protein expression levels of AhR and CYP1A1 of each group were detected by western blotting. (B) In NCI-N87 cells, the protein expression levels of AhR and CYP1A1 of each group were detected by western blotting. ${ }^{* *} \mathrm{P}<0.01$ and ${ }^{* * * *} \mathrm{P}<0.001$ vs. the control. Tax, taxifolin; AhR, aryl hydrocarbon receptor; CYP1A1, cytochrome P450 1A1.

A

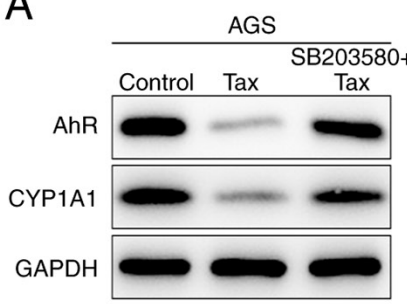

B

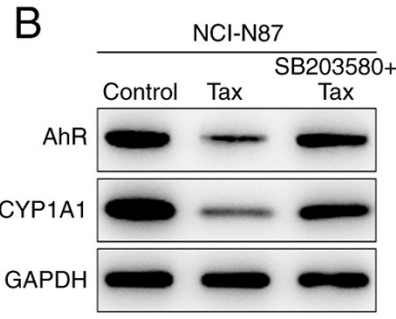

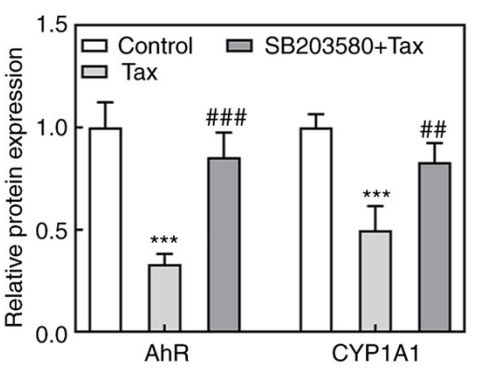

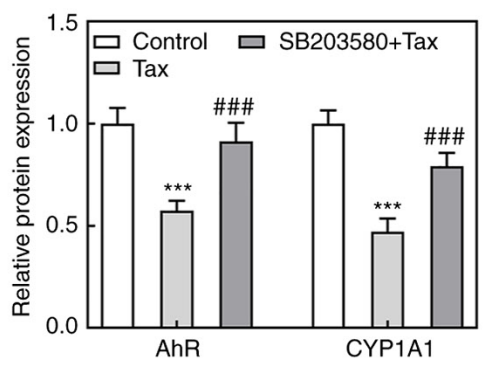

C

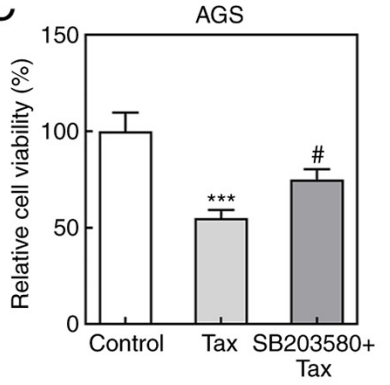

E

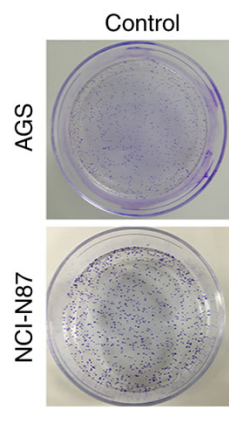

D

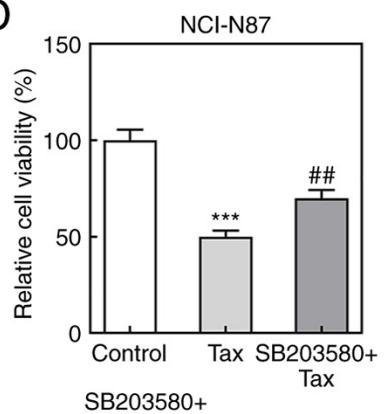

Tax

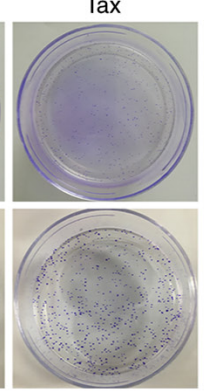
Tax

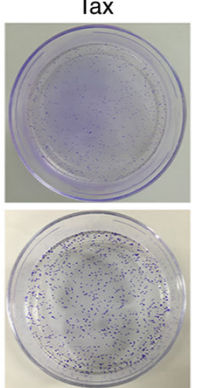

Figure 4. Effects of AhR agonist, SB203580, on cell viability and proliferation in Tax-treated gastric cancer cells. (A and B) AGS and NCI-N87 cells were treated with Tax or co-treated with Tax and SB203580, an agonist of AhR. Then, the protein expression levels of AhR and CYP1A1 were assessed using western blotting and quantified. (C and D) The cell viability was assessed using a Cell Counting Kit-8 assay. (E) Cell colony formation assays were performed. ${ }^{* * *} \mathrm{P}<0.001$ vs. the control; ${ }^{\#} \mathrm{P}<0.05,{ }^{\# \#} \mathrm{P}<0.01$ and ${ }^{\# \# \#} \mathrm{P}<0.001$ vs. Tax. Tax, taxifolin; AhR, aryl hydrocarbon receptor; CYP1A1, cytochrome P450 1A1.

hand, the addition of SB203580 increased the viability of AGS and NCI-N87 cells, and increased the colony formation number compared with Tax treatment alone (Fig. 4C-E). On the other hand, the hindered migratory and invasive abilities of Tax were partly restored by SB203580, which was further verified by the upregulated protein expression levels of MMP2 and MMP9 in the SB203580 + Tax group (Fig. 5A-D). Furthermore, SB203580 diminished the expression levels of E-cadherin and ZO-1, whereas it elevated the expression levels of N-cadherin and Snail in Tax-treated AGS and NCI-N87 cells (Fig. 5E). Therefore, these results indicated that the inhibition of AhR/CYP1A1 signaling partly attenuated the effects of Tax on gastric cancer cells.

Effects of Tax on gastric cancer in vivo. Finally, the antitumor effects of Tax were further examined in vivo. A mouse tumor model was established by injecting AGS or NCI-N87 cells subcutaneously into the right flank region. Following sacrifice, the tumors were removed and weighed. As revealed in Fig. 6A and B, compared with the controls, the size and weight of tumors from mice injected with AGS cells and treated with Tax were significantly decreased. During the process of tumor growth, tumor size was recorded every 3 days. The curve presented in Fig. 6C illustrates a continuous inhibition of tumor size by Tax treatment. The body weights of mice were also monitored every three days, but there were no significant differences between these two groups (Fig. 6D). Similar results were obtained from mice injected with NCI-N87 cells (Fig. 6E-H). These results demonstrated that Tax suppressed the growth of gastric cancer in vivo. In addition, immunohistochemical analysis revealed that Tax treatment greatly reduced the expression level of Ki67 of tumor tissues from mice injected with AGS or NCI-N87 cells (Fig. 7A and B). The protein expression levels of Ki67, PCNA, MMP2 and MMP9 in the tumor tissues were markedly reduced by Tax treatment (Fig. 7C and D). Concurrently, the protein expression levels of E-cadherin and ZO-1 were upregulated, whereas the protein expression levels of $\mathrm{N}$-cadherin and Snail were downregulated 
A
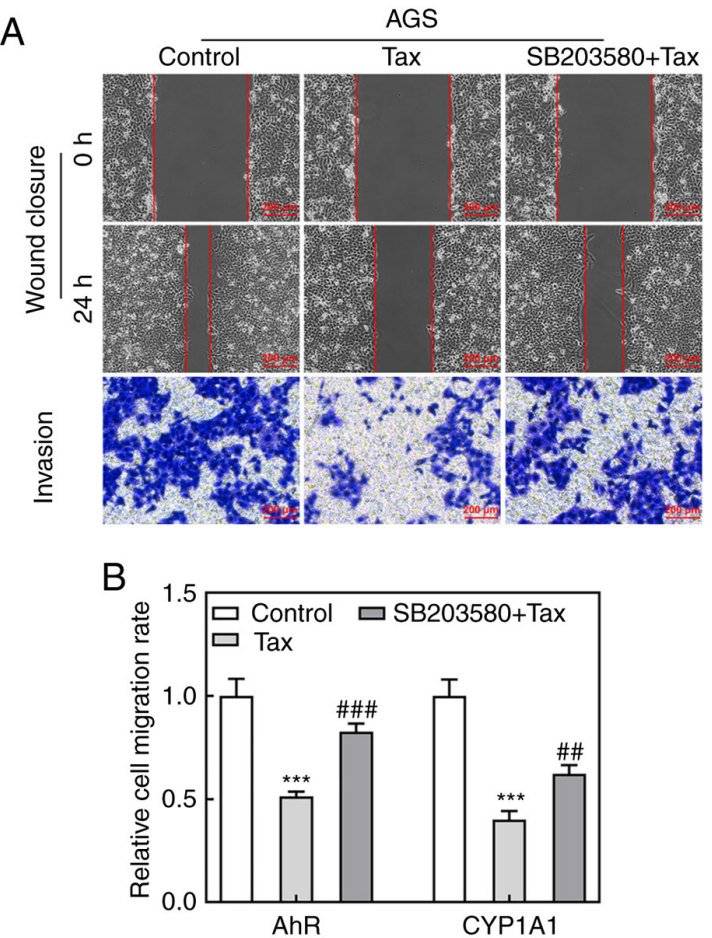

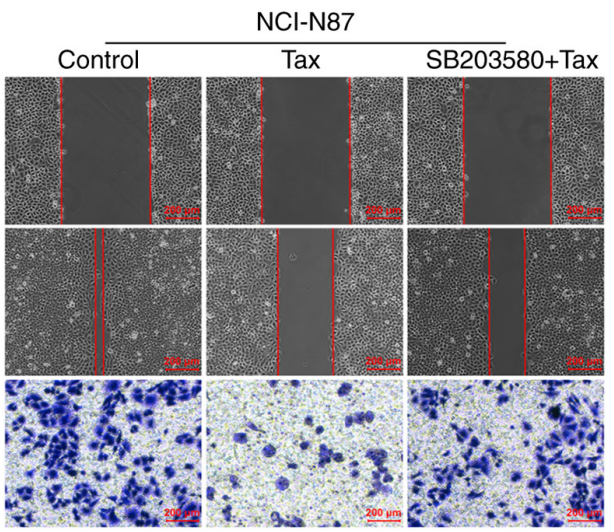

C

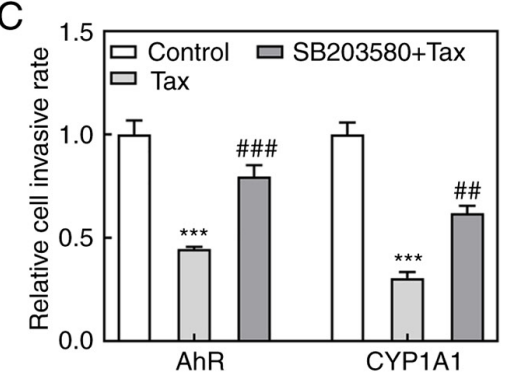

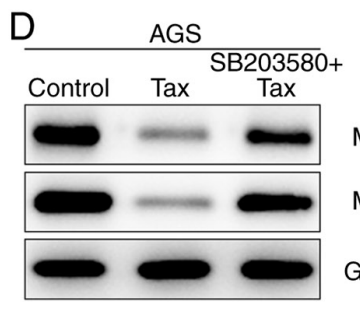

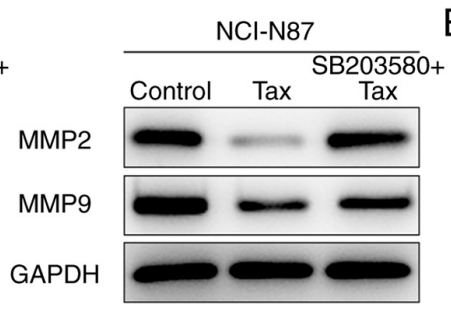

E
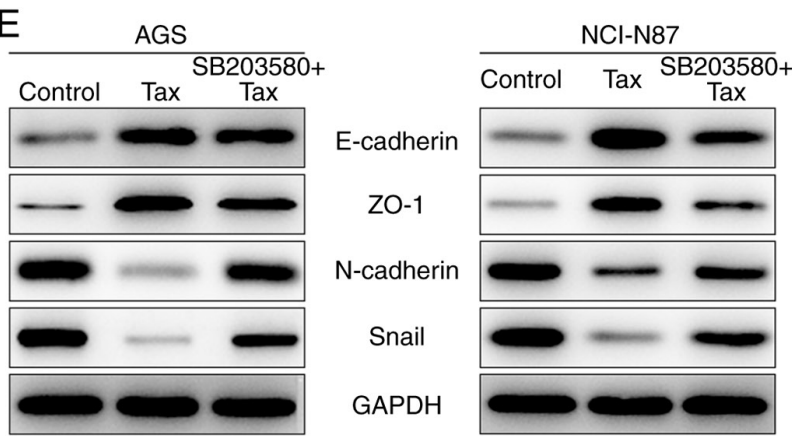

Figure 5. Effects of AhR agonist, SB203580, on cell migration and invasion in Tax-treated gastric cancer cells. (A) Wound-healing and Transwell assays were conducted to observe cell migration and invasion abilities, respectively. (B) The migration rate of each group was quantified. (C) The invasion rate of each group was quantified. (D and E) The protein expression of MMP2, MMP9, E-cadherin, ZO-1, N-cadherin and Snail was assessed by western blotting. ${ }^{* * * *} \mathrm{P}<0.001$ vs. the control; ${ }^{\# /} \mathrm{P}<0.01$ and ${ }^{\# \# \#} \mathrm{P}<0.001$ vs. Tax. Tax, taxifolin; AhR, aryl hydrocarbon receptor; CYP1A1, cytochrome P450 1A1; MMP, matrix metalloproteinase; ZO-1, Zonula occludens-1.

by Tax treatment (Fig. 7C and D); these results were consistent with those obtained in vitro. Furthermore, AhR/CYP1A1 signaling was reduced, as evidenced by the significantly downregulated protein expression levels of AhR and CYP1A1 following Tax treatment (Fig. 7E and F). Therefore, the in vivo experiments further demonstrated the antitumor activity of Tax in gastric cancer and its potential mechanisms of action.

\section{Discussion}

Gastric cancer is one of the most common malignancies and the third leading cause of cancer-related mortality worldwide (11). Patients with gastric cancer are characterized as 'three low and three high', whereby the 5-year survival rate, early diagnosis rate and radical resection are low, and the morbidity, mortality and metastatic rate are high (12). Thus, discovery of effective therapeutics is crucial to overcome this problem. Tax has been reported to possess certain antitumor properties. However, its effects on gastric cancer have not yet been explored, at least to the best of our knowledge.
The inhibition of uncontrolled cell proliferation is crucial to hindering tumor progression. In the present study, it was revealed that Tax inhibited the viability of two gastric cancer cell lines (AGS and NCI-N87) in a concentration-dependent manner. On the basis of these findings, the suitable Tax concentration was used in subsequent experiments to assess the effects of Tax on cellular biological behaviors. The results revealed that Tax significantly reduced cell colony formation, indicating that Tax exerted a suppressive effect on the cell proliferative ability. In addition, Tax significantly diminished the cell migratory and invasive abilities of AGS and NCI-N87 cells. To explore these inhibitory activities in further detail, the protein expression of MMPs and EMT markers was determined. In malignancies, MMPs promote a large range of cellular processes, including cell proliferation, migration and invasion, as well as facilitating EMT (13). During the process of EMT, polarized epithelial cells complete multifaceted changes and acquire mesenchymal cell phenotypes. In particular, the loss of E-cadherin, a marker of epithelial cells, and the gain of $\mathrm{N}$-cadherin, a marker of mesenchymal cells, 
A

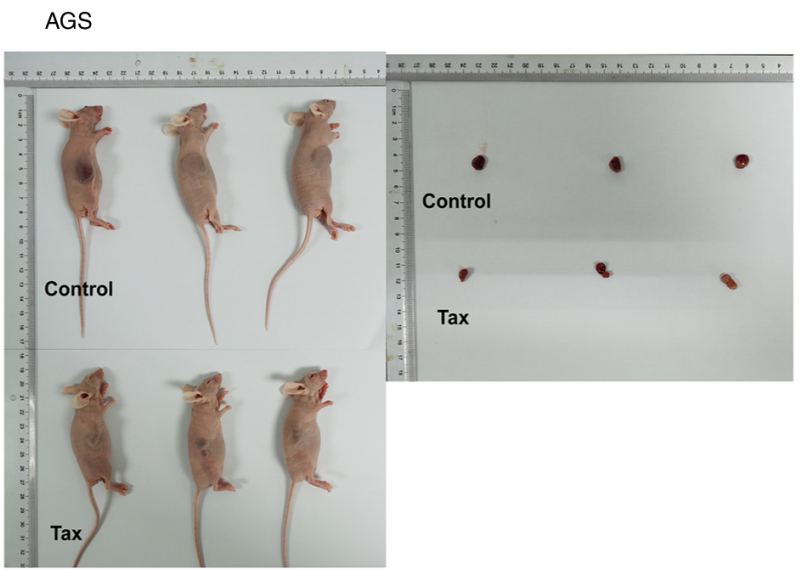

E $\quad$ NCl-N87

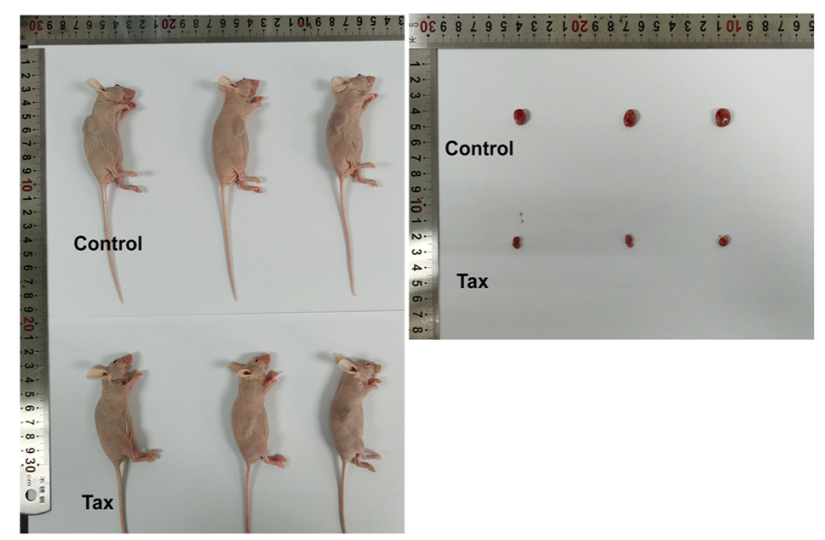

B AGS

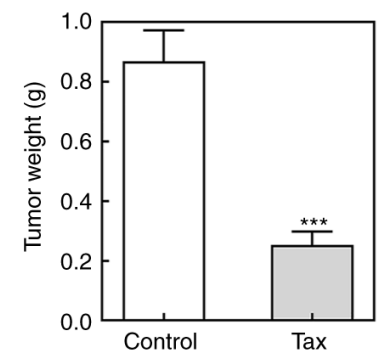

F

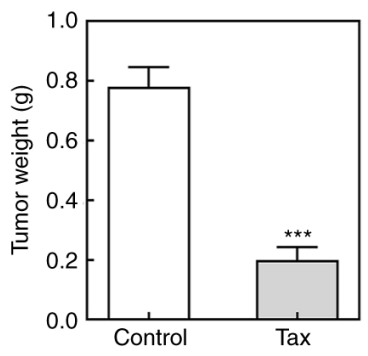

C
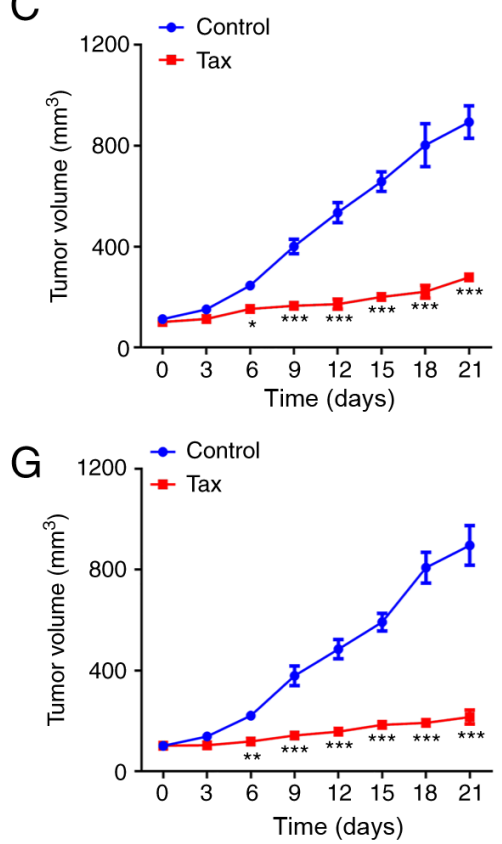

D

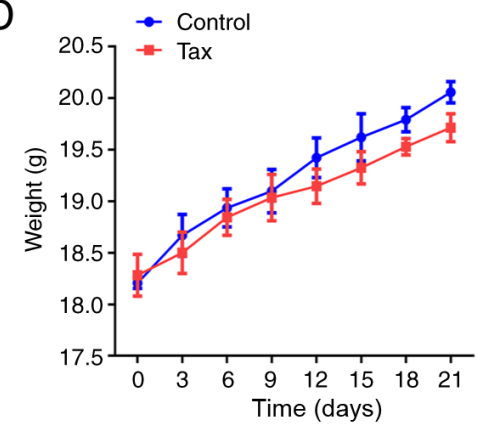

$\mathrm{H}$

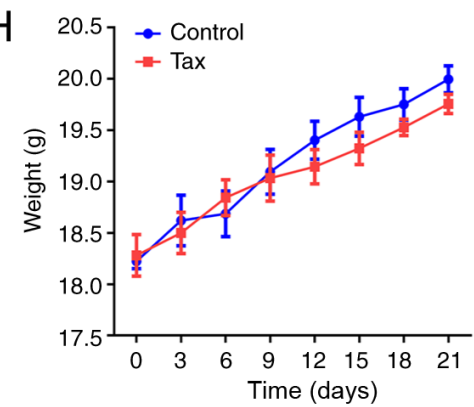

Figure 6. Effects of Tax on tumor growth of gastric cancer in vivo. (A) BALB/c null nude mice were administered AGS cell suspension injection to establish a gastric cancer animal model. After sacrifice, the tumors were removed for observation. (B) The tumor weights were recorded. (C) The tumor volumes during the tumor growth process were recorded every three days. (D) The body weight of mice during the tumor growth process was recorded every three days. (E-H) BALB/c null nude mice were administered NCI-N87 cell suspension injection to establish a gastric cancer animal model. The tumor weights and tumor volumes were recorded. ${ }^{*} \mathrm{P}<0.05,{ }^{* *} \mathrm{P}<0.01$ and ${ }^{* * * *} \mathrm{P}<0.001$. Tax, taxifolin.

are the principal characteristics of EMT (14). Coenzyme Q0 has been reported to inhibit MMP9 expression and increase the expression of E-cadherin in breast cancer tumors, and to prevent tumor cell metastasis (15). Sinulariolide has also been revealed to exhibit antitumor activity in gastric cancer by inhibiting cell migration and invasion through the downregulation of the EMT process (16). In the present study, the upregulation of E-cadherin and ZO-1, and the downregulation of N-cadherin and Snail, were also observed following Tax treatment, indicating that Tax significantly inhibited the EMT process of gastric cancer cells. Thus, Tax exhibited potent antitumor activity in gastric cancer by inhibiting cell proliferation, migration, invasion and EMT.

AhR is a cytosolic ligand-activated transcriptional factor and plays an important role in the regulation of cancer development. Once activated, AhR can initiate the transcriptional regulation of a range of genes, such as CYP1A1, which is involved in chemically-induced carcinogenesis (17-21). Thus, the AhR/CYP1A1 signaling pathway has been widely researched in various types of cancer, and plays a crucial role in the regulation of cancer progression. Al-Dhfyan et al (21) demonstrated that the AhR/CYP1A1 signaling pathway controlled the proliferation, self-renewal ability and chemoresistance of breast cancer stem cells. Maayah et al (19) revealed that the inhibition of the AhR/CYP1A1 pathway exerted protective effects against breast cancer initiation in human epithelial breast cells. Yin et al (22) indicated that AhR and CYP1A1 were upregulated in colorectal cancer tissues and keratinocyte growth factor promoted cell proliferation via AhR signaling in colorectal cancer cells. In addition, it was reported that knockdown of AhR effectively decreased cell proliferation, migration and invasion abilities in gastric cancer cells (23), demonstrating an important regulatory role of AhR/CYP1A1 in gastric cancer; however, the effects of Tax 
A

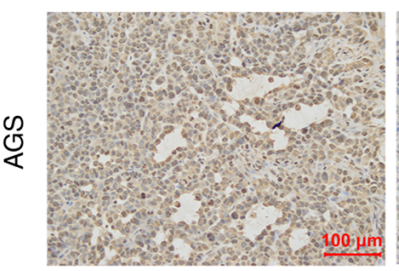

Control

C

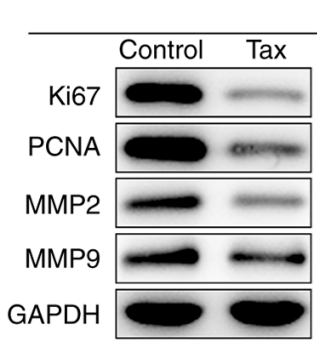

AGS
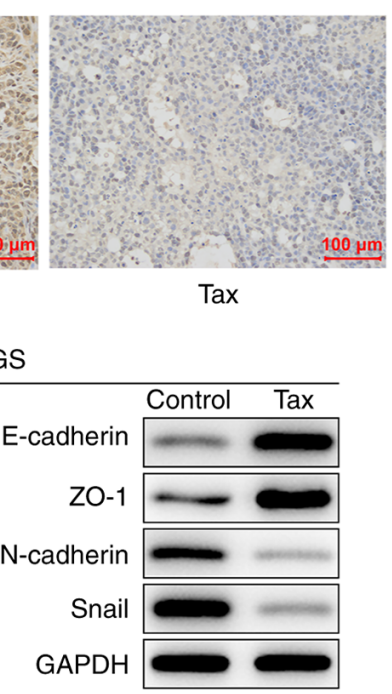

$\mathrm{E}$
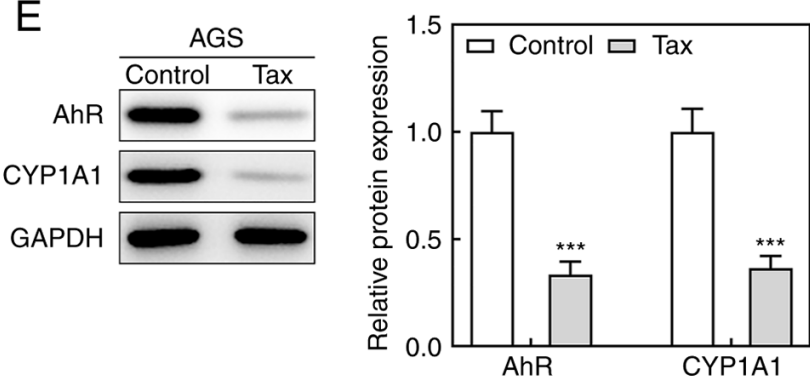

B

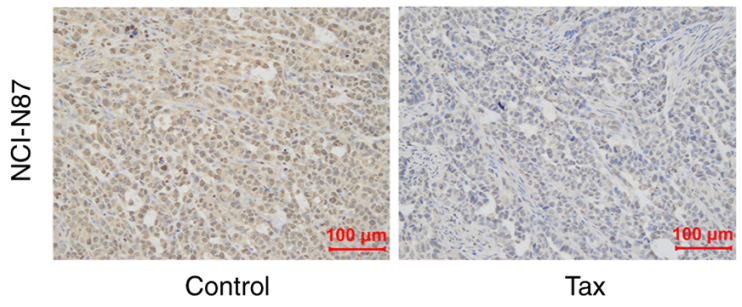

D

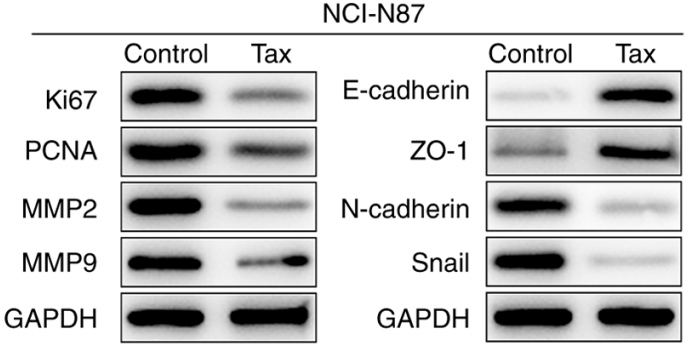

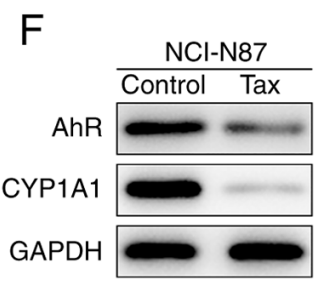

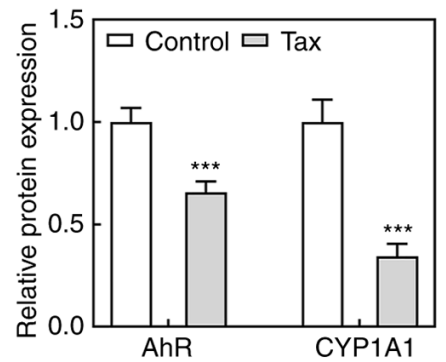

Figure 7. Effects of Tax on tumor growth of gastric cancer in vivo. (A and B) BALB/c null nude mice were administered AGS/NCI-N87 cell suspension injection to establish a gastric cancer animal model, and were treated with Tax. After sacrifice, immunohistochemical analysis was conducted to investigate the expression level of Ki67 of tumor tissues from mice injected with AGS or NCI-N87 cells. (C and D) The protein expression levels of Ki67, PCNA, MMP2, MMP9, E-cadherin, N-cadherin, ZO-1 and Snail were assessed using western blotting. (E and F) The protein expression levels of AhR and CYP1A1 were assessed using western blotting. ${ }^{* * *} \mathrm{P}<0.001$ vs. the control. Tax, taxifolin; PCNA, proliferating cell nuclear antigen; MMP, matrix metalloproteinase; ZO-1, Zonula occludens-1; AhR, aryl hydrocarbon receptor; CYP1A1, cytochrome P450 1A1.

on the AhR/CYP1A1 signaling pathway in gastric cancer have not been reported thus far, and whether Tax exerted its antitumor function through the AhR/CYP1A1 signaling pathway in gastric cancer remains unclear, and requires clarification. Of note, a recent study demonstrated that Tax inhibited breast carcinogenesis by inhibiting the AhR/CYP1A1 signaling pathway (24). Thus, it was hypothesized that Tax may exert its antitumor effects through AhR/CYP1A1 signaling pathway in gastric cancer. As was anticipated, Tax treatment reduced the protein expression levels of AhR and CYP1A1 in gastric cancer in vitro and in vivo. SB203580 was then used to activate AhR/CYP1A1 signaling. Further experiments revealed that the activation of AhR/CYP1A1 signaling partly abolished the suppressive effects of Tax on gastric cancer cell proliferation, migration and invasion, indicating that Tax exerted its antitumor effects partly via inhibiting the AhR/CYP1A1 signaling pathway.

However, certain limitations remain to be addressed in the present study. Firstly, the potential mechanism of Tax in gastric cancer via the AhR/CYP1A1 signaling pathway was focused on, and the results revealed that the antitumor effects of Tax in gastric cancer could be partly abolished by SB203580, an AhR agonist; however, knocking down AhR and CYP1A1 in mice is also a direct experiment which is necessary to be conducted in the future to verify the critical role of AhR/CYP1A1 signaling underlying the protective role of Tax in gastric cancer. Secondly, RNA-sequencing is another effective way to verify our conclusion or further analyze the mechanism of Tax in gastric cancer. In addition, RNA-sequencing will provide much information about the differential genes before or after Tax treatment, which is necessary to be further investigated in our future work.

In conclusion, the present study determined for the first time, to the best of our knowledge, that Tax inhibited gastric cancer cell proliferative, migratory and invasive abilities, and inhibited tumor growth. In addition, Tax exerted its antitumor effects partly by inhibiting the AhR/CYP1A1 signaling pathway. These findings provide a promising strategy for the treatment of gastric cancer.

\section{Acknowledgements}

Not applicable.

\section{Funding}

The present study was supported by the Cooperative Fund of Nanchong Government and North Sichuan Medical College (grant no. 18SXHZ0357) and the Scientific fund of North Sichuan Medical College (grant no. CBY15-A-ZD008). 


\section{Availability of data and materials}

All data generated or analyzed during this study are included in this published article.

\section{Authors' contributions}

XW conceptualized and designed the study. JX and YP performed the experiments and acquired the data. XW and JX analyzed and interpreted the data. All authors wrote the manuscript. All authors read and approved the final manuscript.

\section{Ethics approval and consent to participate}

All animal experiments were performed in accordance with the Care and Use of Laboratory Animals established by the US National Institutes of Health and were approved by the Ethics Committee of West China Hospital, Sichuan University (Chengdu, China).

\section{Patient consent for publication}

Not applicable.

\section{Competing interests}

The authors declare that they have no competing interests.

\section{References}

1. Smyth EC, Nilsson M, Grabsch HI, van Grieken NC and Lordick F: Gastric cancer. Lancet 396: 635-648, 2020.

2. Bray F, Ferlay J, Soerjomataram I, Siegel RL, Torre LA and Jemal A: Global cancer statistics 2018: GLOBOCAN estimates of incidence and mortality worldwide for 36 cancers in 185 countries. CA Cancer J Clin 68: 394-424, 2018.

3. Song Z, Wu Y, Yang J, Yang D and Fang X: Progress in the treatment of advanced gastric cancer. Tumour Biol 39: $1010428317714626,2017$.

4. Sunil C and Xu B: An insight into the health-promoting effects of taxifolin (dihydroquercetin). Phytochemistry 166: 112066, 2019.

5. Li J, Hu L, Zhou T, Gong X, Jiang R, Li H, Kuang G, Wan J and Li H: Taxifolin inhibits breast cancer cells proliferation, migration and invasion by promoting mesenchymal to epithelial transition via $\beta$-catenin signaling. Life Sci 232: 116617, 2019.

6. Razak S, Afsar T, Ullah A, Almajwal A, Alkholief M, Alshamsan A and Jahan S: Taxifolin, a natural flavonoid interacts with cell cycle regulators causes cell cycle arrest and causes tumor regression by activating Wnt/ $\beta$-catenin signaling pathway. BMC Cancer 18: 1043, 2018.

7. Wang R, Zhu X, Wang Q, Li X, Wang E, Zhao Q, Wang Q and $\mathrm{Cao} \mathrm{H}$ : The anti-tumor effect of taxifolin on lung cancer via suppressing stemness and epithelial-mesenchymal transition in vitro and oncogenesis in nude mice. Ann Transl Med 8: 590, 2020.

8. Chen X, Gu N, Xue C and Li BR: Plant flavonoid taxifolin inhibits the growth, migration and invasion of human osteosarcoma cells. Mol Med Rep 17: 3239-3245, 2018.
9. Percie du Sert N, Hurst V, Ahluwalia A, Alam S, Avey MT, Baker M, Browne WJ, Clark A, Cuthill IC, Dirnagl U, et al: The ARRIVE guidelines 2.0: Updated guidelines for reporting animal research. PLoS Biol 18: e3000410, 2020.

10. National Research Council (US): Committee for the Update of the Guide for the Care and Use of Laboratory Animals: Guide for the Care and Use of Laboratory Animals. National Academies Press, Washington, DC, 2011.

11. Torre LA, Bray F, Siegel RL, Ferlay J, Lortet-Tieulent J and Jemal A: Global cancer statistics, 2012. CA Cancer J Clin 65: 87-108, 2015.

12. Wu H, Wang W, Tong $\mathrm{S}$ and $\mathrm{Wu} \mathrm{C}$ : Nucleostemin regulates proliferation and migration of gastric cancer and correlates with its malignancy. Int J Clin Exp Med 8: 17634-17643, 2015.

13. Scheau C, Badarau IA, Costache R, Caruntu C, Mihai GL, Didilescu AC, Constantin C and Neagu M: The role of matrix metalloproteinases in the epithelial-mesenchymal transition of hepatocellular carcinoma. Anal Cell Pathol (Amst) 2019: 9423907, 2019.

14. Lamouille S, Xu J and Derynck R: Molecular mechanisms of epithelial-mesenchymal transition. Nat Rev Mol Cell Biol 15: 178-196, 2014.

15. Yang HL, Thiyagarajan V, Shen PC, Mathew DC, Lin KY, Liao JW and Hseu YC: Anti-EMT properties of CoQ0 attributed to PI3K/AKT/NFKB/MMP-9 signaling pathway through ROS-mediated apoptosis. J Exp Clin Cancer Res 38: 186, 2019.

16. Wu YJ, Lin SH, Din ZH, Su JH and Liu CI: Sinulariolide inhibits gastric cancer cell migration and invasion through downregulation of the EMT process and suppression of FAK/PI3K/AKT/mTOR and MAPKs signaling pathways. Mar Drugs 17: 668, 2019.

17. Kamenickova A and Dvorak Z: Effects of flavored mineral waters on AhR-CYP1A1 signaling pathway in primary human hepatocytes and in human hepatic and intestinal cancer cells. Food Chem Toxicol 50: 1933-1939, 2012.

18. Haarmann-Stemmann T, Abel J, Fritsche E and Krutmann J: The AhR-Nrf2 pathway in keratinocytes: On the road to chemoprevention? J Invest Dermatol 132: 7-9, 2012.

19. Maayah ZH, Ghebeh H, Alhaider AA, El-Kadi AO, Soshilov AA, Denison MS, Ansari MA and Korashy HM: Metformin inhibits 7,12-dimethylbenz[a]anthracene-induced breast carcinogenesis and adduct formation in human breast cells by inhibiting the cytochrome P4501A1/aryl hydrocarbon receptor signaling pathway. Toxicol Appl Pharmacol 284: 217-226, 2015.

20. Vondracek J, Umannova L and Machala M: Interactions of the aryl hydrocarbon receptor with inflammatory mediators: Beyond CYP1A regulation. Curr Drug Metab 12: 89-103, 2011.

21. Al-Dhfyan A, Alhoshani A and Korashy HM: Aryl hydrocarbon receptor/cytochrome P450 1A1 pathway mediates breast cancer stem cells expansion through PTEN inhibition and beta-Catenin and Akt activation. Mol Cancer 16: 14, 2017.

22. Yin J, Sheng B, Pu A, Han B, Yang K, Wang Q, Sun L and Yang $\mathrm{H}$ : Keratinocyte growth factor regulation of aryl hydrocarbon receptor activation in colorectal cancer cells. Dig Dis Sci 61: 444-452, 2016.

23. Yin XF, Chen J, Mao W, Wang YHand Chen MH: Downregulation of aryl hydrocarbon receptor expression decreases gastric cancer cell growth and invasion. Oncol Rep 30: 364-370, 2013.

24. Haque MW and Pattanayak SP: Taxifolin inhibits 7,12dimethylbenz(a)anthracene-induced breast carcinogenesis by regulating AhR/CYP1A1 signaling pathway. Pharmacogn Mag 13 (Suppl 4): S749-S755, 2018.

This work is licensed under a Creative Commons Attribution-NonCommercial-NoDerivatives 4.0 International (CC BY-NC-ND 4.0) License. 